\section{Here today, there tomorrow}

The benefits of digital impressioning are by now widely acknowledged throughout the dental profession. But the Mobile True Definition Scanner from 3M Oral Care takes these advantages to a whole new level.

Building on the success of the cart system - which is proven to be one of the most accurate intraoral scanners on the market - the mobile version affords simple portability. This means you only need one
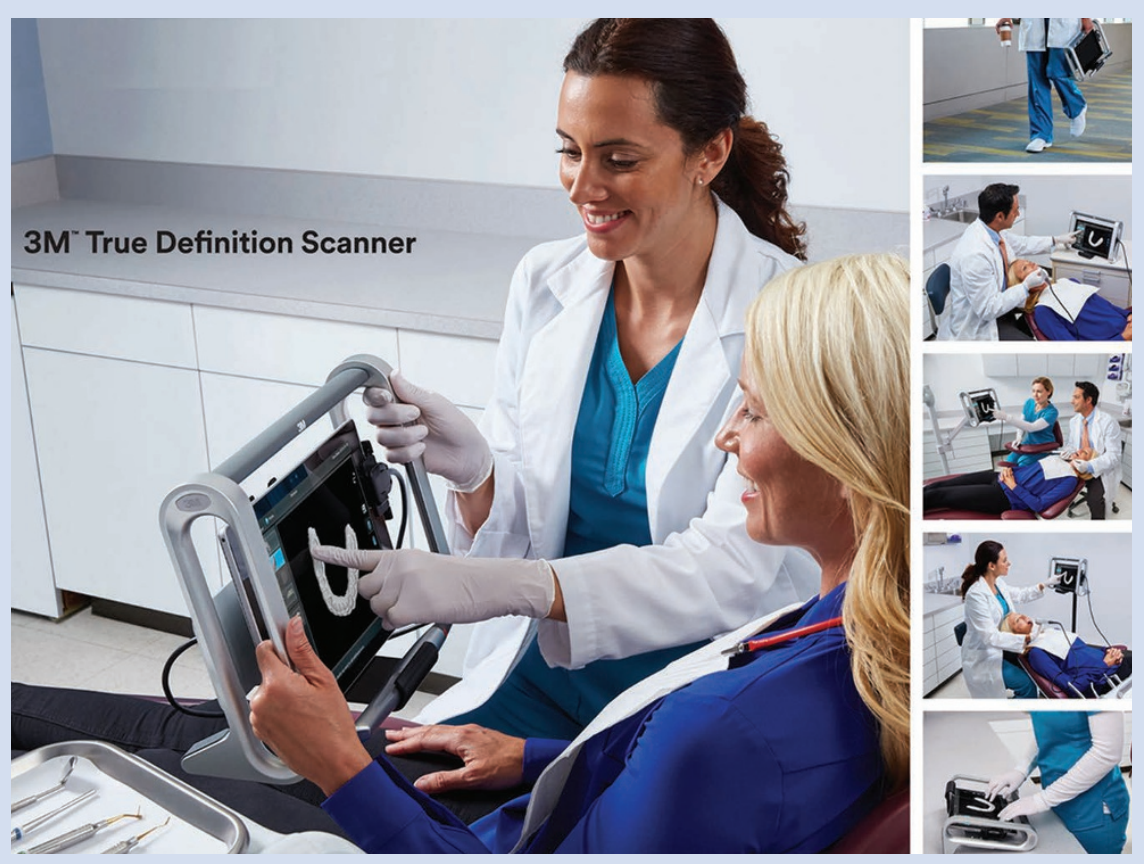

\section{Beating bruxism}

Dental Sky are now the exclusive distributor of the Flexi Orthotic System (F.O.S.).

The F.O.S. offers chairside treatment to reduce and eliminate your patients' headaches, migraines and other associated head and neck pain caused by the clenching or grinding of teeth (bruxism). The objective of the F.O.S. is to allow muscles in spasm to relax, protect the teeth and jaws from the adverse effects of bruxism, and allow normalisation of the periodontal ligament proprioception.

This revolutionary and unique design comprises of a choice of either an upper or lower anterior splint made of medical grade polyester copolymer. The material is heat adjustable and can be directly

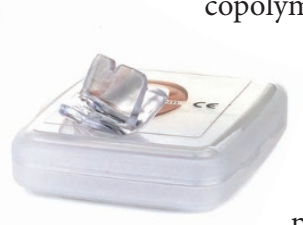
bonded to, allowing the treatment to be long wearing without any discoloration, risk of bacteria or damage caused by separation of materials. The system will continue to work after many years of use without any problems for you or your patient, reducing chair time and excessive patient recalls.

This system is quick and easy to fit chairside in just 15 minutes, or alternatively impressions can be sent to Dental Sky's lab where their technicians will make the splint for you. With every purchase of a starter pack you will receive a complimentary training course from one of Dental Sky's experienced technicians as well as full support and aftercare.

For more information or to order yours, visit https://www.dentalsky.com/flexi-orthoticsystem-starter-kit-fos.html.

\section{Probe with precision}

Transform the way you detect and diagnose periodontal disease with the innovative Florida Probe.

\section{Available exclusively from Clark}

Dental, the Florida Probe system will act as your computerised assistant in periodontal examinations, charting as you probe. The system's constant-force probe, with a precision of $0.2 \mathrm{~mm}$, maintains consistent measurements among examiners and improves the accuracy of readings for more precise diagnostics.

Charting can be customised to feature various details, including pocket depth, recession, bleeding and suppuration. You

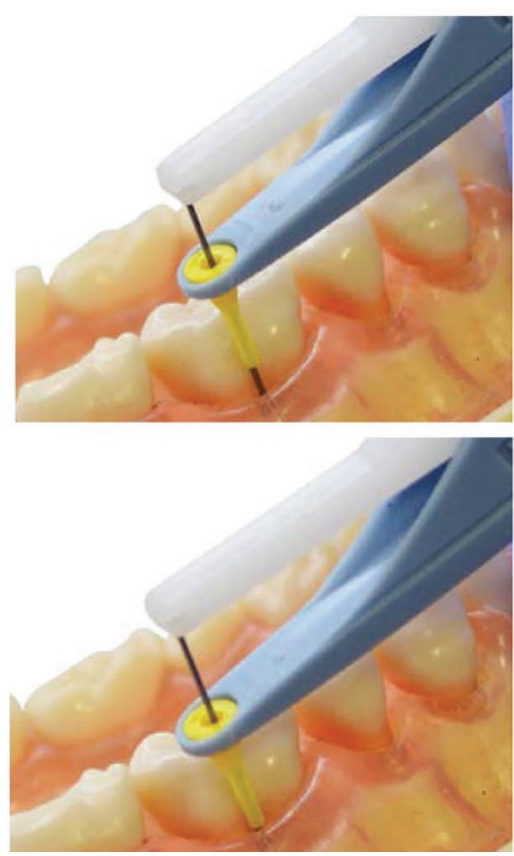

can retain peace of mind in the knowledge that the system also provides exceptional clinical and legal documentation.

The true value of the Florida Probe lies in its ability to increase patient acceptance of periodontal therapies through its all-inclusive, understandable system. The 'talking probe' calls out numbers and messages as you work, making patient communication quicker, simpler and more effective.

Consider the Florida Probe for your periodontal examinations. Contact the expert team at Clark Dental to find out more.

For more information call Clark Dental on 01268733 146, email info@clarkdental. co.uk or visit www.clarkdental.co.uk. 